\title{
Infinitely many hypermaps of a given type and genus
}

\author{
Gareth A. Jones \\ School of Mathematics, University of Southampton \\ Southampton SO17 1BJ, UK \\ G.A.Jones@maths.soton.ac.uk \\ Daniel Pinto \\ CMUC, Department of Mathematics, University of Coimbra \\ 3001-454 Coimbra, Portugal \\ dpinto@mat.uc.pt \\ Submitted: Jul 20, 2010; Accepted: Jul 29, 2010; Published: Nov 5, 2010 \\ Mathematics Subject Classification: 05C10, 05C25, 20E07

\begin{abstract}
It is conjectured that given positive integers $l, m, n$ with $l^{-1}+m^{-1}+n^{-1}<1$ and an integer $g \geqslant 0$, the triangle group $\Delta=\Delta(l, m, n)=\langle X, Y, Z| X^{l}=Y^{m}=Z^{n}=$ $X Y Z=1\rangle$ contains infinitely many subgroups of finite index and of genus $g$. A slightly stronger version of this conjecture is as follows: given positive integers $l$, $m, n$ with $l^{-1}+m^{-1}+n^{-1}<1$ and an integer $g \geqslant 0$, there are infinitely many nonisomorphic compact orientable hypermaps of type $(l, m, n)$ and genus $g$. We prove that these conjectures are true when two of the parameters $l, m, n$ are equal, by showing how to construct appropriate hypermaps.
\end{abstract}

\section{Introduction}

The following conjecture arose in discussions with Jürgen Wolfart:

Conjecture $1.1(\mathrm{~A})$. Given positive integers $l, m, n$ with $l^{-1}+m^{-1}+n^{-1}<1$, and an integer $g \geqslant 0$, the triangle group

$$
\Delta=\Delta(l, m, n)=\left\langle X, Y, Z \mid X^{l}=Y^{m}=Z^{n}=Z Y Z=1\right\rangle
$$

contains infinitely many subgroups of finite index and of genus $g$.

The well-known connections between triangle groups and hypermaps (discussed in [4], for example), yield the following slightly stronger form of this conjecture (see section 3): 
Conjecture $1.2(\mathrm{~B})$. Given positive integers $l, m, n$ with $l^{-1}+m^{-1}+n^{-1}<1$, and an integer $g \geqslant 0$, there are infinitely many nonisomorphic compact orientable hypermaps of type $(l, m, n)$ and of genus $g$.

In these conjectures, which are independent of the ordering of $l, m$ and $n$, it is necessary to impose the inequality to avoid trivial cases. The natural action of $\Delta$ is on the Riemann sphere, the complex plane or the hyperbolic plane as $l^{-1}+m^{-1}+n^{-1}>1,=1$ or $<1$. In the first case, $\Delta$ is finite and there are only finitely many hypermaps of a given type $(l, m, n)$, all of them having genus 0 . In the second case, $\Delta$ is abelian-by-finite and there are infinitely many subgroups and hypermaps of genus 0 or 1 , but none of any genus $g>1$. We will therefore assume from now on that we are in the third case, where the triple $(l, m, n)$ is said to be hyperbolic.

The conjectures are false if one restricts attention to uniform hypermaps (equivalently torsion-free subgroups of $\Delta$ ), those for which the hypervertices, hyperedges and hyperfaces all have valencies $l, m$ and $n$ respectively; this includes the case of regular hypermaps, corresponding to normal subgroups of $\Delta$. The reason is that in this case the size of the hypermap (equivalently the index of the corresponding subgroup) is proportional to its Euler characteristic, so for a fixed genus there can be only finitely many uniform hypermaps of a given type. We shall therefore allow nonuniform hypermaps, where the valencies of the hypervertices, hyperedges and hyperfaces have least common multiples $l, m$ and $n$ respectively, but they are not necessarily all equal to $l, m$ and $n$. Our main result is the following:

Theorem 1.1. Conjectures $A$ and $B$ are true in all cases where at least two of $l, m$ and $n$ are equal.

Hypermaps of type $(l, 2, n)$ are simply maps of type $\{n, l\}$ in the notation of Coxeter and Moser [2], where we interpret this more widely to mean that the valencies of the faces and the vertices have least common multiples $n$ and $l$. Such a type is hyperbolic provided $l^{-1}+n^{-1}<\frac{1}{2}$, or equivalently $(l-2)(n-2)>4$. We therefore have:

Corollary 1.1. Conjecture $B$ is true for maps of each type $\{n, n\}$ with $n \geqslant 5$.

Our method of proof of Theorem 1 (from section 7 onwards) is take $l=m$ and to construct the required hypermaps of type $(m, m, n)$ by first constructing their Walsh bipartite maps [9]. These are maps of type $\{2 n, m\}$ and genus $g$, so (with a change of notation and applying duality) our method of proof yields:

Corollary 1.2. Suppose that either $m$ or $n$ is even. Then Conjecture $A$ is true for $\Delta(2, m, n)$ and Conjecture $B$ is true for maps of type $\{m, n\}$.

The representation of a hypermap by its Walsh bipartite map corresponds to the inclusion of $\Delta(m, m, n)$ as a subgroup of index 2 in $\Delta(m, 2,2 n)$ (see [4] for this and other representations of hypermaps). Similar arguments, based on triangle group inclusions described by Singerman in [7], imply: 
Corollary 1.3. Conjecture $A$ is true for $\Delta(2,3,7)$ and $\Delta(2,3,9)$, and Conjecture $B$ is true for maps of type $\{3,7\}$ and $\{3,9\}$.

These results leave many remaining cases in which Conjectures A and B are still open, for instance for maps of type $\{m, n\}$ where $m$ and $n$ are odd, excluding those types covered by Corollary 1.3. Indeed, in many cases it is not clear whether there are any hypermaps of a given type and genus, let alone infinitely many.

\section{Hypermaps and triangle groups}

The connections between hypermaps and triangle groups are described in some detail in [4], but for convenience we will summarise them here, mainly in the case of orientable hypermaps without boundary. The extended triangle group

$$
\Delta[l, m, n]=\left\langle R_{0}, R_{1}, R_{2} \mid R_{i}^{2}=\left(R_{1} R_{2}\right)^{l}=\left(R_{2} R_{0}\right)^{m}=\left(R_{0} R_{1}\right)^{n}=1\right\rangle
$$

is generated by reflections $R_{0}, R_{1}$ and $R_{2}$ in the sides of a triangle $T$ with angles $\pi / l, \pi / m$ and $\pi / n$ in a simply connected Riemann surface $\mathcal{U}$, where $\mathcal{U}$ is the Riemann sphere, the complex plane or the hyperbolic plane as $l^{-1}+m^{-1}+n^{-1}>1,=1$ or $<1$. The orientation-preserving subgroup of index 2 in $\Delta[l, m, n]$ is the triangle group

$$
\Delta=\Delta(l, m, n)=\left\langle X, Y, X \mid X^{l}=Y^{m}=Z^{n}=X Y Z=1\right\rangle,
$$

generated by rotations $X=R_{1} R_{2}, Y=R_{2} R_{0}$ and $Z=R_{0} R_{1}$ through angles $2 \pi / l, 2 \pi / m$ and $2 \pi / n$ around the vertices of $T$. These two groups are the full automorphism group and the orientation-preserving automorphism group of the universal hypermap $\tilde{\mathcal{H}}$ of type $\tau=(l, m, n)$ drawn on $\mathcal{U}$. Any hypermap $\mathcal{H}$ of this type is isomorphic to the quotient of $\tilde{\mathcal{H}}$ by some subgroup $H \leqslant \Delta[l, m, n]$, which is unique up to conjugacy. Conversely, any conjugacy class of subgroups $H$ determines a hypermap $\mathcal{H} / H$ of type $\tau^{\prime}=\left(l^{\prime}, m^{\prime}, n^{\prime}\right)$ where $l^{\prime}, m^{\prime}$ and $n^{\prime}$ (dividing $l, m$ and $n$ ) are the orders of the permutations of the cosets of $H$ induced by $X, Y$ and $Z$. Two hypermaps are isomorphic if and only if the corresponding subgroups are conjugate in $\Delta[l, m, n]$ (or in $\Delta(l, m, n)$ if we require an orientationpreserving isomorphism). Compact hypermaps $\mathcal{H}$ correspond to subgroups $H$ of finite index in $\Delta[l, m, n]$, and those on orientable surfaces without boundary correspond to subgroups $H \leqslant \Delta(l, m, n)$.

Any subgroup $H$ of finite index in $\Delta$ has a presentation

$$
\begin{gathered}
H=\left\langle a_{1}, b_{1}, \ldots, a_{g}, b_{g}, x_{1}, \ldots, x_{r}, y_{1}, \ldots, y_{s}, z_{1}, \ldots, z_{t}\right| \\
\left.\prod_{i=1}^{g}\left[a_{i}, b_{i}\right] \cdot \prod_{i=1}^{r} x_{i} \cdot \prod_{i=1}^{s} y_{i} \cdot \prod_{i=1}^{t} z_{i}=x_{i}^{l_{i}}=y_{i}^{m_{i}}=z_{i}^{n_{i}}=1\right\rangle,
\end{gathered}
$$

where $g \geqslant 0$ and each $l_{i}, m_{i}$ or $n_{i}$ is a nonidentity divisor of $l, m$ or $n$ respectively. Here $g$, called the genus of $H$, is the genus of the corresponding hypermap $\mathcal{H}$, and the generators 
$x_{i}, y_{i}$ and $z_{i}$ correspond to any cycles of $X, Y$ and $Z$ of lengths $l / l_{i}<l, m / m_{i}<m$ or $n / n_{i}<n$ in their action on the cosets of $H$ in $\Delta$, or equivalently to any degenerate hypervertices, hyperedges and hyperfaces of $\mathcal{H}$, those of valencies $l / l_{i}<l, m / m_{i}<m$ or $n / n_{i}<n$. We say that $H$ has signature $\left(g ; l_{1}, \ldots, l_{r}, m_{1}, \ldots, m_{s}, n_{1}, \ldots, n_{t}\right)$. These parameters are related by the Riemann-Hurwitz formula

$$
2 g-2+\sum_{i=1}^{r}\left(1-\frac{1}{l_{i}}\right)+\sum_{i=1}^{s}\left(1-\frac{1}{m_{i}}\right)+\sum_{i=1}^{t}\left(1-\frac{1}{n_{i}}\right)=N\left(1-\frac{1}{l}-\frac{1}{m}-\frac{1}{n}\right),
$$

where $N=|\Delta: H|$. The hypermap $\mathcal{H}$ is uniform if and only if $r=s=t=0$, or equivalently $H$ is a surface group, with signature $(g ;-)$.

A permutation of the triple $(l, m, n)$ corresponds to a renaming of the generators of $\Delta[l, m, n]$ and of $\Delta(l, m, n)$, or equivalently to one of Machì's operations on hypermaps, permuting hypervertices, hyperedges and hyperfaces [5]. We can therefore identify $\Delta(l, m, n)$ with $\Delta\left(l^{\prime}, m^{\prime}, n^{\prime}\right)$ for any permutation $\left(l^{\prime}, m^{\prime}, n^{\prime}\right)$ of the triple $(l, m, n)$.

Hypermaps of type $(l, 2, n)$ are equivalent to maps of type $\{n, l\}$, where we interpret this notation more generally than in [2] to mean that the valencies of the faces and the vertices have least common multiples $n$ and $l$.

\section{The relationship between Conjecture A and Con- jecture B}

Suppose that Conjecture B is true for a given triple $\tau=(l, m, n)$ and a given genus $g$, so that there are infinitely many nonisomorphic hypermaps $\mathcal{H}$ of type $\tau$ and genus $g$. These correspond to mutually nonconjugate subgroups $H$ of finite index in $\Delta=\Delta(l, m, n)$, all of genus $g$, so Conjecture $\mathrm{A}$ is true for $\tau$ and $g$.

Conversely, suppose that Conjecture A is true for type $\tau$ and genus $g$, so that $\Delta$ has infinitely many subgroups $H$ of genus $g$. Having finite index, each $H$ has only finitely many conjugates, so among these subgroups there are infinitely many which are mutually nonconjugate, corresponding to infinitely many nonisomorphic hypermaps $\mathcal{H}$ of genus $g$. Each of these has type $\tau^{\prime}=\left(l^{\prime}, m^{\prime}, n^{\prime}\right)$ for some divisors $l^{\prime}, m^{\prime}$ and $n^{\prime}$ of $l, m$ and $n$, namely the orders of the permutations induced by $X, Y$ and $Z$ on the cosets of $H$. For a given triple $\tau$ there are only finitely many such triples $\tau^{\prime}$, so for at least one of them — but not necessarily for $\tau$ itself — there must be infinitely many nonisomorphic hypermaps of type $\tau^{\prime}$ and genus $g$. In particular, if $g>1$ then this type must be hyperbolic. In this situation, it is conceivable that there could be only finitely many hypermaps of type $\tau$ and genus $g$ (or even none), though we know of no example of this phenomenon.

This shows that Conjecture $\mathrm{A}$ is a weaker statement than Conjecture B. We will therefore first prove Conjecture B for various triples $\tau$ and genera $g$, so that we can immediately deduce Conjecture A for the same $\tau$ and $g$. The following result shows that for a given type $\tau$, it is in fact sufficient to prove Conjecture B for genera $g=0,1$ and 2 . 
Theorem 3.1. Suppose that there are infinitely many nonisomorphic hypermaps of type $\tau$ and genus 2 , and that $G$ is a 2-generator group of order $g-1$ for some $g \geqslant 2$. Then there are infinitely many nonisomorphic hypermaps $\mathcal{K}$ of type $\tau$ and genus $g$ with $G \leqslant$ Aut $\mathcal{K}$.

Proof. Let $\mathcal{H}$ be an orientable hypermap of type $\tau$ and genus 2. This corresponds to a subgroup $H \leqslant \Delta$ as described in the preceding section. By mapping the generators $a_{1}$ and $a_{2}$ of $H$ to a pair of generators for $G$, and all the other canonical generators of $H$ to the identity, we obtain an epimorphism $H \rightarrow G$. The kernel $K$ is a normal subgroup of index $g-1$ in $H$, corresponding to a hypermap $\mathcal{K}$ of type $\tau$ and genus $g$, which is a regular unbranched $(g-1)$-sheeted covering of $\mathcal{H}$ with covering group $G \cong H / K \leqslant$ Aut $\mathcal{K}$. Since each hypermap $\mathcal{K}$ can arise in this way from only finitely many hypermaps $\mathcal{H}$, the result follows.

In each case one can, for example, take $G$ to be a cyclic group of the appropriate order, so this reduces the problem of proving Conjecture B to the cases $g=0,1$ and 2 . One can also reduce the proof of Conjecture B for genus 1 to that of constructing a single hypermap of type $\tau$ and genus 1 :

Theorem 3.2. If $\mathcal{H}$ is a hypermap of type $\tau$ and genus 1 , and $G$ is any 2-generator finite abelian group, then there is a hypermap $\mathcal{K}$ of type $\tau$ and genus 1 which is a regular unbranched covering of $\mathcal{H}$ with covering group $G$.

Proof. The argument is similar to that used for Theorem 3.1, except that now the generators $a_{1}$ and $b_{1}$ of $H$ are mapped to a pair of generators of $G$, and the rest to the identity.

In particular, by taking $G$ to be arbitrarily large we see that if there is at least one hypermap of type $\tau$ and genus 1 then there are infinitely many.

Although these two theorems apparently reduce the task of proving Conjecture $\mathrm{B}$ for any given type, a direct proof of the result for all $g$ in fact uses exactly the same ingredients as a proof of the results for $g \leqslant 2$. One should therefore regard these theorems as giving slightly stronger results, rather than as reducing the task of proving them.

\section{The general method}

In proving Theorem 1, we will construct each hypermap $\mathcal{H}$ by first constructing its Walsh map $\mathcal{W}=W(\mathcal{H})[9]$. This is a bipartite map on the same surface as $\mathcal{H}$, with each hypervertex or hyperedge of $\mathcal{H}$ represented as a black or white vertex, each incidence between them represented as an edge between the corresponding vertices, so that each vertex has the same valency as the hypervertex or hyperedge it represents, and each hyperface of $\mathcal{H}$ represented as a face of twice the valency (since it is bordered by alternating black and white vertices).

When assuming that two of $l, m$ and $n$ are equal, we may by permuting them assume that $l=m$, so that we are dealing with hypermaps $\mathcal{H}$ of type $\tau=(m, m, n)$. These 
correspond to bipartite maps $\mathcal{W}=\mathcal{W}(\mathcal{H})$ of type $\{2 n, m\}$ on the same surface, with a colour-preserving isomorphism $\mathcal{W}(\mathcal{H}) \cong \mathcal{W}\left(\mathcal{H}^{\prime}\right)$ if and only if $\mathcal{H} \cong \mathcal{H}^{\prime}$, so if Conjecture $\mathrm{B}$ is true for hypermaps of type $\tau$ then it is also true for maps of type $\mu=\{2 n, m\}$. Equivalently, we are using the inclusion of $\Delta(m, m, n)$ as a subgroup of index 2 in $\Delta(m, 2,2 n)$ to deduce Conjecture A for the latter group from its truth for the first group.

In order to prove Conjecture $\mathrm{B}$ for a specific triple $\tau=(m, m, n)$ we will construct bipartite maps $\mathcal{W}$ of type $\mu=\{2 n, m\}$ by joining together suitable numbers of copies of a few basic 'building blocks'. These are bipartite maps $\mathcal{A}=\mathcal{A}_{\mu}, \mathcal{T}=\mathcal{T}_{\mu}$ and $\mathcal{D}=\mathcal{D}_{\mu}$ on three surfaces with boundary, namely a closed annulus $A$, a torus minus two open discs, called 2-trisc and denoted by $T$, and a closed disc $D$. Sometimes, we will use $\mathcal{A}_{i}$, $\mathcal{T}_{i}$ and $\mathcal{D}_{i}$ (with $i=n$ or $m$, instead of $\mu$ ) when we just want to pay attention to the valencies of the faces or to the valencies of the vertices (assuming the other parameter of $\mu$ is fixed and known). We will give the precise details of the construction of these building blocks later, starting in section 7. By taking suitably many copies of them, and joining them in pairs by identifying boundary components, compact orientable bipartite maps $\mathcal{W}=\mathcal{W}_{\mu}$ of type $\mu$ and of arbitrary genus $g$ can be constructed, and these are the Walsh maps $\mathcal{W}(\mathcal{H})$ of the required hypermaps $\mathcal{H}$. For this to work, one has to ensure that the interior of each building block 'looks like' part of a bipartite map of type $\mu$, and that the boundary identifications produce suitable local behaviour, so that the final result is a bipartite map of this type.

To ensure this, we will construct the maps $\mathcal{A}, \mathcal{T}$ and $\mathcal{D}$ so that each of their boundary components $C$ is a cycle in the map, homeomorphic to $S^{1}$ and consisting of vertices and edges. We define an allowed joining of two such maps to be an identification of a pair of their boundary components $C_{0}$ and $C_{1}$ by means of a homeomorphism $C_{0} \rightarrow C_{1}$ which matches vertices with vertices of the same colour, so that $C_{0}$ and $C_{1}$ become a single cycle in the resulting bipartite map. If vertices of valencies $v_{0}$ and $v_{1}$ in $C_{0}$ and $C_{1}$ are identified with each other, they give rise to a vertex of valency $v=v_{0}+v_{1}-2$, so we also require that $v$ divides $m$; in fact, we will generally arrange that $v=m$.

If two surfaces $X_{0}$ and $X_{1}$ are joined by identifying their boundary components $C_{0}$ and $C_{1}$, then the resulting surface has Euler characteristic $\chi\left(X_{0} \cup X_{1}\right)=\chi\left(X_{0}\right)+\chi\left(X_{1}\right)$. Now $\chi(A)=0, \chi(T)=-2$ and $\chi(D)=1$, so if $g \geqslant 2$ then $g-1$ copies of $\mathcal{B}$ and an arbitrary number $h \geqslant 0$ of copies of $\mathcal{A}$ can be joined pairwise in some cyclic order to give an orientable bipartite map $\mathcal{W}$ of characteristic $2-2 g$ and hence of genus $g$; by fixing $g$ and letting $h$ vary we obtain the required infinite set of nonisomorphic hypermaps $\mathcal{H}$. Alternatively, it is sufficient to take one copy of $\mathcal{T}$ and an arbitrary number of copies of $\mathcal{A}$, giving infinitely many hypermaps of genus 2 , and then by using Theorem 3.1 to extend this to any genus $g>2$. Similarly, if $g=1$ we can take $h$ copies of $\mathcal{A}$ in cyclic order, where $h \geqslant 1$ (or just one copy if we use Theorem 3.2), and if $g=0$ we can use $h$ copies of $\mathcal{A}$ in linear order, with the two ends of the resulting tube capped by copies of $\mathcal{D}$.

If we ignore the vertex-colours, we can regard each $\mathcal{W}=\mathcal{W}_{\mu}$ as an orientable map of type $\mu$, so these constructions prove Conjecture B for maps of this type, and hence prove Conjecture A for the corresponding triangle group $\Delta(m, 2,2 n)$. With a minor change of notation, this proves Corollary 1.2. 
This method of proof is based on that used in [3], where similar building blocks were used to construct infinitely many maps of type $\{3,24\}$ for each genus $g \geqslant 0$, and then the corresponding subgroups of $\Delta(24,2,3)$ were lifted back via the natural epimorphism $\Delta(\infty, 2,3) \rightarrow \Delta(24,2,3)$ to obtain infinitely many noncongruence subgroups of genus $g$ in the modular group $\Delta(\infty, 2,3)=P S L_{2}(\mathbf{Z})$.

\section{$5 \quad$ Proof of Corollary 1.3}

Singerman [7] has classified all pairs of hyperbolic triangle groups $\Delta=\Delta(l, m, n)$ and $\Delta^{\prime}=\Delta\left(l^{\prime}, m^{\prime}, n^{\prime}\right)$ such that $\Delta$ is a subgroup of $\Delta^{\prime}$ (necessarily of finite index). The list includes several infinite families, such as $\Delta(s, s, t) \leqslant \Delta(2, s, 2 t)$ with index 2 , and finitely many sporadic examples, such as $\Delta(7,7,7) \leqslant \Delta(2,3,7)$ with index 24 and $\Delta(9,9,9) \leqslant$ $\Delta(2,3,9)$ with index 12 .

Given such an inclusion $\Delta \leqslant \Delta^{\prime}$, any subgroup $H$ of genus $g$ in $\Delta$ is automatically a subgroup of genus $g$ in $\Delta^{\prime}$, so if Conjecture $\mathrm{A}$ is true for $\Delta$ then it is also true for $\Delta^{\prime}$. In particular, the inclusions $\Delta(7,7,7) \leqslant \Delta(2,3,7)$ and $\Delta(9,9,9) \leqslant \Delta(2,3,9)$, together with Theorem 1.1, show that $\Delta(2,3,7)$ and $\Delta(2,3,9)$ satisfy Conjecture A, thus proving the first part of Corollary 1.3.

If $\Delta \leqslant \Delta^{\prime}$ then the hypermap $\mathcal{H}$ corresponding to an inclusion $H \leqslant \Delta$ gives rise to a hypermap $\mathcal{H}^{\prime}$ of the same genus corresponding to the inclusion $H \leqslant \Delta^{\prime}$. Since $\Delta$ has finite index in $\Delta^{\prime}$, at most finitely many conjugacy classes of subgroups $H$ in $\Delta$ can lie in the same conjugacy class in $\Delta^{\prime}$, so this function $\mathcal{H} \mapsto \mathcal{H}^{\prime}$ is finite-to-one on isomorphism classes; it follows that any infinite set of nonisomorphic hypermaps $\mathcal{H}$ gives rise to infinitely many nonisomorphic hypermaps $\mathcal{H}^{\prime}$. In general, there is no guarantee that these hypermaps $\mathcal{H}^{\prime}$ will have type $\left(l^{\prime}, m^{\prime}, n^{\prime}\right)$. However, in the two cases we are interested in, namely $\Delta(7,7,7) \leqslant \Delta(2,3,7)$ and $\Delta(9,9,9) \leqslant \Delta(2,3,9)$, the canonical generators of $\Delta^{\prime}$ of orders 2,3 and 7 or 9 induce permutations of these orders on the cosets of $\Delta$, and hence also on the cosets of any subgroup $H \leqslant \Delta$, so $\mathcal{H}^{\prime}$ has type $(2,3,7)$ or $(2,3,9)$ respectively. Thus Theorem 1.1 implies that Conjecture B is true for hypermaps of these two types, and hence for maps of types $\{3,7\}$ and $\{3,9\}$. This proves the second part of Corollary 1.3.

Similar arguments can be applied to various other triangle group inclusions, such as $\Delta(4,8,8) \leqslant \Delta(2,3,8)$, but the results obtained are particular cases of Corollary 1.2. It is also possible to give direct proof of Corollary 1.3 for $\Delta(2,3,7)$, either by designing Lego pieces to build maps of type $\{3,7\}$ or by deducing it from results of Stothers [8] on subgroups of this triangle group. Since the periods 2, 3 and 7 are prime, a subgroup $H$ of finite index in $\Delta(2,3,7)$ must have signature $\sigma=\left(g ; 2^{(r)}, 3^{(s)}, 7^{(t)}\right)$ for some integers $g, r, s, t \geqslant 0$. Stothers used coset diagrams to show that for all but finitely many choices of $g, r, s$ and $t$ there is a subgroup $H$ of finite index with the corresponding signature $\sigma$. In particular, by fixing $g$ and letting $r, s$ and $t$ vary we obtain Corollary 1.3 for this group. 


\section{Multiplication of an edge}

Some of the methods will be applied, with small modifications, several times. One of the operations that will often be used is the multiplication of an edge $e$ of the map, by an integer $k$, and that consists of replacing $e$ with $k$ edges between the same pair of vertices, enclosing $k-1$ new faces of valency 2. If $e$ is a boundary edge then one of these new edges will also be a boundary edge (but not the other ones).

The valencies of the vertices of the boundary components are relevant to describe the pieces and to confirm that a map of a specific type is obtained when they are glued together. We say that a boundary component (denoted by $\partial^{i} A, \partial^{i} T$ or $\partial D$ for $i=0,1$ ) has type $k^{(t)}$ if it has $t$ vertices of valency $k$. If the vertices have not all the same valency, we will explicitly give those different valencies to the reader.

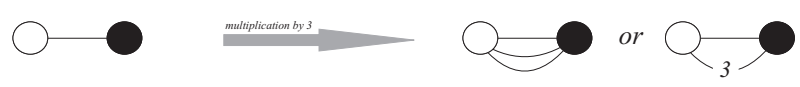

Figure 1: Multiplication of an edge by 3.

Important note: We leave, in the drawing of the graph, the edge that is multiplied. It follows that that edge should not be counted twice. For instance, the number 3, in Figure 1, means exactly the number of edges between those two vertices.

\section{The proof}

We will divide the proof into several cases for different families of hypermaps. There will be three different main cases:

i) when $n$ is even and the parameters are not too small (if they are not $\leqslant 3$ );

ii) when $n$ is odd and the parameters are not too small (if they are not $\leqslant 4$ );

iii) the other possibilities, when at least one of the parameters is small.

All possibilities will be covered but we will solve the problem by dealing, in the following order, with families of hypermaps of type:

- $(m, m, n)$ with $m \geqslant 4$, even $n \geqslant 4$;

- $(m, m, 2)$ with $m \geqslant 6$;

- $(5,5,2)$;

- $(3,3,4)$; 
- $(3,3, n)$ with even $n \geqslant 6$;

- $(m, m, n+1)$ with $m \geqslant 5$, odd $n+1 \geqslant 5$;

- $(m, m, 3)$ with $m \geqslant 5$;

- $(4,4,3)$;

- $(4,4, n)$ with, odd $n \geqslant 5$;

- $(3,3, n)$ with odd $n \geqslant 5$.

\section{Hypermaps of type $(m, m, n)$ with $n$ even}

When proving Theorem 1.1, we may without loss of generality assume that $l=m$. In considering hypermaps of type $\tau=(m, m, n)$ we will first deal with the case where $n$ is even. The Walsh maps $\mathcal{W}$ have type $\mu=\{2 n, m\}$, so their vertices and faces must have valencies dividing $m$ and $2 n$ respectively; we will, in fact, construct each bipartite map $\mathcal{W}$ so that all its vertices have valency $m$, and the face-valencies (which are necessarily even) are equal to 2,4 or $2 n$, corresponding to hyperfaces of valencies 1,2 or $n$.

\subsection{Hypermaps of type $(m, m, n)$ with $m \geqslant 4$, even $n \geqslant 4$}

For each even $n$, let $\mathcal{R}_{n}$ be a bipartite map on the rectangle $[0,4] \times[0,2 n-6] \subset \mathbb{R}^{2}$.

This bipartite map (see Figure 2) has vertices at the points: $(0, j),(1, j)$, for $i \in$ $\{n-3, \ldots, 2 n-6\} \cup\{0\} ;(2, j),(3, j)$, for $j \in\{0, \ldots, n-3\} \cup\{2 n-6\} ;(4, j)$, for $j \in$ $\{n-3,2 n-6\} \cup\{0\}$. The vertices $(i, j)$ are black or white if $i+j$ is even or odd, respectively. Because we want some of them to be adjacent, we introduce some edges: the horizontal edges $(i, j) \times(i, j+1)$ for $i \in\{0, n-3,2 n-6\}$ and $j \in\{0, \ldots, 3\} ;(i, 0) \times$ $(i, 1)$ for $i \in\{n-2, \ldots, 2 n-7\},(i, 2) \times(i, 3)$ for $i \in\{1, \ldots, n-4\}$; and vertical edges $(i, j) \times(i+1, j)$ for $i \in\{n-3, \ldots, 2 n-7\}$ and $j \in\{0,1,4\}(i, j) \times(i+1, j)$ for $i \in\{0, \ldots, n-$ $4\}$ and $j \in\{2,3\}$. These edges enclose $2 n-4$ faces. $2 n-6$ of them are square faces: $0<x<1, \quad j<y<j+1$ for $j \in\{n-3, \ldots, 2 n-7\} ; 2<x<3, \quad j<y<j+1$ for $j \in$ $\{0, \ldots, n-4\}$; and two of them are 2n-gons: $0<x<2$ or $3<x<4$, and $0<y<n-3$ $1<x<4$ and $n-3<y<2 n-6$.

To obtain a bipartite map on the torus, we identify the opposite sides in the usual way: $(4, y)=(0, y)$ for $0 \leqslant y \leqslant 2 n-6$ and $(x, 2 n-6)=(x, 0)$ for $0 \leqslant x \leqslant 4$. All the vertices have valency 3 at this stage. To build a 2 -trisc $\mathcal{T}$ we need to remove two discs. We can do this by removing two non adjacent square faces (see Figure 3 ). For instance: $0<x<1, \quad n-3<y<n-2$ and $2<x<3, \quad n-4<y<n-3$.

The trivalent map on the 2 -trisc, $\mathcal{T}_{n}$, has now $2 n-8$ square faces and two $2 n$-gonal faces. The two boundary components of $\mathcal{T}_{n}$ have both type $3^{(4)}$ (they have 4 vertices of valency 3). We will use this bipartite map as a basis to build blocks of type $\mu=$ 


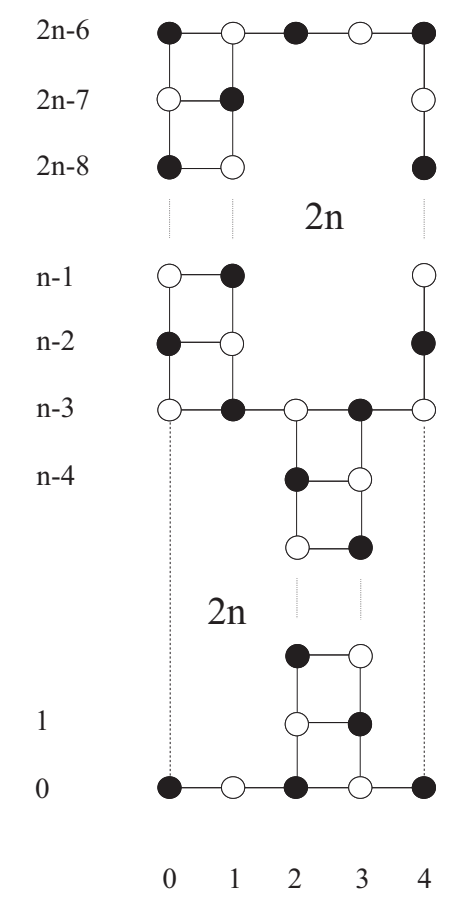

Figure 2: Bipartite map on the rectangle $[0,4] \times[0,2 n-6]$.

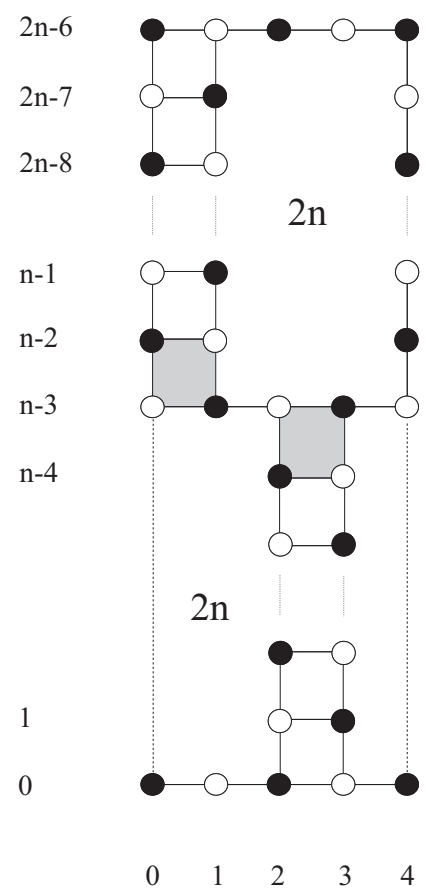

Figure 3: 2-trisc. 
$\{2 n, m\}$. These are obtained by multiplying by $m-2$ each horizontal edge of the form: $(i, 0) \times(i, 1)$ for $i \in\{n-1, \ldots, 2 n-6\}$ and $(i, 2) \times(i, 3)$ for $i \in\{0, \ldots, n-5\}$.

Then we choose integers $m_{0}, m_{1} \geqslant 3$ such that $m_{0}+m_{1}=m+2$ and, for each $i=0,1$, we multiply each of the horizontal edges in the boundary components $\partial^{i} \mathcal{T}_{\mu}$ by $m_{i}-2$. This is a general procedure that always works but we could fix (for instance) $m_{0}=3$ and then take $m_{1}=m-1$, multiplying only the edges of one of the boundary components of $\partial^{i} \mathcal{T}_{\mu}$.

Each vertex is incident with exactly one of these multiplied edges. Therefore, every internal vertex has valency $m$ and the vertices on the boundary component $\partial^{i} \mathcal{T}_{\mu}(\mathrm{i}=0,1)$ have valency $m_{i}$, so that this component has type $m_{i}^{(4)}$.

Hence, this modified map on the 2-trisc (with some edges multiplied) has two faces of valency $2 n$ and $2 n-8$ faces of valency 4 , just as the first basic bipartite map we have built on this surface, but also: $2(m-3)(n-4)+2\left(m_{0}-3\right)+2\left(m_{1}-3\right)=2(n-3)(m-3)$ new faces of valency 2 . This does not affect the type of the hypermap since they correspond to hyperfaces of valency 1 in the hypermap.

The bipartite map on the annulus is constructed using the same tessellation $\mathcal{R}_{n}$, identifying, as before, the left and right sides but not the top and bottom sides. We obtain, by this process, a map $\mathcal{A}_{\mu}$ with two faces of valency $2 n$ and $2 n-6$ faces of valency 4. The two boundary components $\partial^{i} \mathcal{A}_{\mu}(i=0,1)$ are cycles of length 4 , like those in $\partial^{i} \mathcal{T}_{\mu}$. If we multiply suitable edges, as before, we can create a bipartite map on $\mathcal{A}$ with all internal vertices of valency $m$, one boundary component of type $m_{0}^{(4)}$ and the other one of type $m_{1}^{(4)}$. This new map has two faces of valency $2 n, 2 n-6$ faces of valency 4 , and the others of valency 2 .

To build the disc for each integer $k \geqslant 2$, we construct a tessellation $\mathcal{D}_{k}$ of a closed disc $\mathcal{D}$, with boundary type $k^{(4)}$. We achieve that by starting with a square, regarded as a bipartite map on $\mathcal{D}$ with one face and with four vertices and four edges on $\partial \mathcal{D}$. Then, we multiply a pair of opposite edges by $k-2$, introducing $2(k-3)$ extra faces of valency 2 , so that all four vertices have valency $k$.

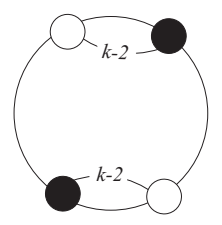

Figure 4: Disc $D_{k}$

The gluing process is now easy to describe. For a given genus $g$, we choose an arbitrarily large $h \in \mathbb{N}_{0}$ and if $g \geqslant 1$ we take $g-1$ copies of $\mathcal{T}$ and $h$ copies of $\mathcal{A}$ in some arbitrary cyclic order. By making allowed joinings between consecutive pieces, we will get a bipartite map $\mathcal{W}_{g, h}$ of genus $g$ and with all vertices of valency $m$. This map $\mathcal{W}_{g, h}$ has $2(g-1+h)$ faces of valency $2 n$, two on each copy of $\mathcal{T}$ or $\mathcal{A}$ and the remaining faces have valency 2 or 4 . Hence, $W_{g, h}$ is the Walsh map of a compact orientable hypermap $\mathcal{H}_{g, h}$ of genus $g$ 
and type $\mu=(m, m, n)$. Because $h$ is as large as we want, we can build in this way an infinite number of nonisomorphic hypermaps of genus $g$ and type $\mu$, as required. If $g=0$ we do not need to use a 2-trisc, we only need $\mathcal{A}$ and two discs $\mathcal{D}_{m_{0}}$ and $\mathcal{D}_{m_{1}}$ (remember $m_{0}+m_{1}=m+2$ ), capping a tube of $h \geqslant 1$ copies of $\mathcal{A}$ in linear order, by allowing joining at its ends. The resulting map will have $2 h$ faces of valency $2 n$ and all other faces of valency 2 or 4 . Since all the vertices have valency $m$, the map is a Walsh bipartite map of a hypermap of type $\mu=(m, m, n)$ on the sphere.

\subsection{Hypermaps of type $(m, m, 2)$ with $m \geqslant 6$}

The method used in the previous case does not work for $n=2$ but we just need to introduce a slight modification to make it right, provided $m \geqslant 6$. This is achieved by using, first, eight $1 \times 1$ square faces to form a tessellation $\mathcal{R}_{2}$ of the rectangle $[0,4] \times[0,2] \subset \mathbb{R}^{2}$ with vertices at the points $(i, j)$ colored black or white as $i+j$ is even or odd. By identifying opposite sides of $\mathcal{R}_{2}$ we obtain a bipartite map of type $\{4,4\}$ on a torus. To build a bipartite map $\mathcal{T}_{2}$ on a 2 -trisc we remove, before identification, two nonadjacent faces: $0<x<1, \quad 0<y<1$ and $2<x<3, \quad 0<y<1$.

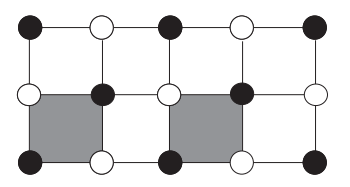

Figure 5: Tessellation of $\mathcal{R}_{2}=[0,4] \times[0,2]$ with 2 faces removed.

This bipartite map has six square faces and each of the eight vertices lies on a boundary component $\partial^{i} \mathcal{T}_{2} \quad(i=0,1)$ of type $4^{(4)}$. Then we choose integers $m_{i} \geqslant 4 \quad(i=0,1)$ so that $m_{0}+m_{1}=m-2$ and multiply each of the two horizontal edges on $\partial^{i} \mathcal{T}_{2}$ by $m_{i}-3$ so that $\partial^{i} \mathcal{T}_{2}$ has type $m_{i}^{(4)}$.

The annulus $\mathcal{A}_{2}$ can be constructed using the same rectangle $\mathcal{R}_{2}$ but only identifying the vertical sides (not the horizontal ones). On each boundary component $\partial^{i} \mathcal{A}_{2} \quad(i=0,1)$ of $\mathcal{A}_{2}$ we multiply each of two nonadjacent edges by $m_{i}-2$ so that this component has type $m_{i}^{(4)}$. Because we now have internal vertices, we also need to multiply each of two nonadjacent internal edges by $m-3$. This will transform all the internal vertices into vertices of valency $m$, as required. With these pieces (together with the discs previously described) we can construct infinitely many hypermaps of type $(m, m, 2)$, provided $m \geqslant 6$.

\subsection{Hypermaps of type $(5,5,2)$}

The method described in the previous subsection does not work for $m=5$ because we have $m_{i} \geqslant 4$ and, consequently, $m=m_{0}+m_{1}-2 \geqslant 6$. To get a hypermap that will work in this particular case, we need to build different blocks whose boundary components have types $3^{(4)}$ and $4^{(4)}$, so that after joining them we will get vertices of valency $3+4-2=5$. 


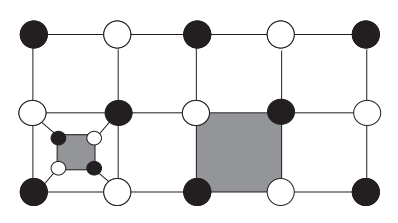

Figure 6: 2-trisc map to build hypermaps of type $(5,5,2)$.

To build $\mathcal{T}$ we use the same bipartite torus map $\mathcal{T}_{2}$ described for the previous case but with four extra vertices, a square $S$, with vertices at $\left(\frac{1}{3}, \frac{1}{3}\right),\left(\frac{2}{3}, \frac{1}{3}\right),\left(\frac{2}{3}, \frac{2}{3}\right)$ and $\left(\frac{1}{3}, \frac{2}{3}\right)$, each joined by a straight edge to the vertex $(0,0),(1,0),(1,1)$ or $(0,1)$ respectively (see Figure 6). This tessellation has five new faces and the new vertices have valency 3 . If we remove the face within $S$, we create a boundary component of type $3^{(4)}$. Removing the face given by $2<x<3$ and $0<y<1$, we create another boundary component, this one of type $4^{(4)}$.

We also need to build an annulus satisfying the same conditions, that is, with one boundary component of type $3^{(4)}$ and another one with type $4^{(4)}$. This can be done in the following way: we take the map of the cube on the sphere, removing a pair of opposite faces and we multiply a pair of opposite edges of one of those faces by 2 . The resulting bipartite map has four faces of valency 4 and two of valency 2 . We just need now two other blocks $\mathcal{D}_{3}$ and $\mathcal{D}_{4}$, as described earlier, and proceed as before but this time joining boundary components of type $3^{(4)}$ to boundary components of type $4^{(4)}$.

\subsection{Hypermaps of type $(3,3,4)$}

The previous methods used square tessellations of a rectangle $\mathcal{R}_{2}$ and can not be applied to build hypermaps of type $(3,3,4)$. The reason is obvious: if we want to obtain, after joining two blocks, a hypermap with hypervertices of valency $m=m_{0}+m_{1}-2$ we will need here $m=3$. So, $m_{0}+m_{1}=5$, giving $m_{0}=2$ and $m_{1}=3$ or vice versa, which is impossible using the strategy we have already introduced. Another method is needed to solve the problem, which means we have to build the blocks following a different idea, an alternative approach.

To build the map $\mathcal{T}$ we take the regular map $\{3,4+4\}$ of type $\{3,8\}$ and genus 2 (described in [2, Chapter 8] and represented in Figure 7, with opposite sides of the octagon identified) and then cut it along a simple closed curve that follows two edges. The map $\{3,4+4\}$, a double cover of the octahedron branched over six vertices, can be constructed by taking a regular octagon, placing vertices at the center, the eight corners and the midpoints of the eight sides; each of these last eight vertices is then joined by straight edges to the central vertex, to the two corner vertices incident with its side, and to the vertices at the midpoints to the two adjacent sides. so that the octagon is tessellated by 16 triangles. If we make orientable identifications of the four pairs of opposite sides of the octagon we obtain the regular map $\{3,4+4\}$. If we identify just three pairs of opposite 
sides instead (or, equivalently, we cut the map $\{3,4+4\}$ open along the simple closed path corresponding to the fourth pair), we obtain a triangular map on a 2-trisc.

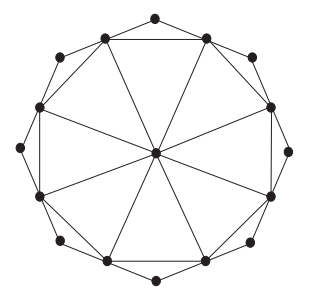

Figure 7: Map $\{3,4+4\}$ of type $\{3,8\}$ and genus 2 .

The boundary components in this block both have type $5^{(2)}$ and all the four interior vertices have valency 8 . To construct the annulus we take $\mathcal{A}=\{z \in \mathbb{C}|1 \leqslant| z \mid \leqslant 2\}$, with vertices at $\pm 1, \pm 2$ and $\pm 3 i / 2$, and with edges along the boundary components, along $\mathcal{A} \cap \mathbb{R}$, and joining $\pm 3 i / 2$ each to \pm 1 and \pm 2 . We have then eight triangular faces and the two internal vertices have valency 4 . The disc is formed by dividing the closed unit disc $D$ into four triangular faces, with vertices at \pm 1 and $\pm i / 2$ and edges along the boundary, along $D \cap \mathbb{R}$ and joining each of $\pm i / 2$ to \pm 1 , so that the internal vertices have valency 2 . In both of these maps, each boundary component has type $5^{(2)}$.

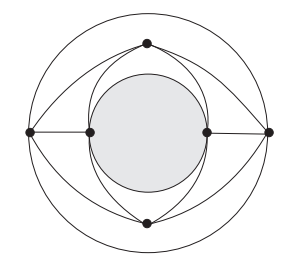

Figure 8: Annulus.

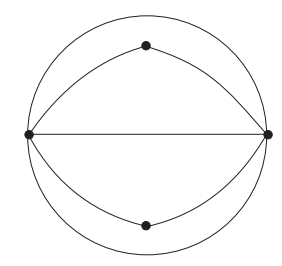

Figure 9: Disc.

All the vertices in the boundary components have valency 5 , which means that after joining together the pieces these vertices will give rise to vertices of degree $8=5+5-2$. Moreover, all the vertices that are not in the boundary have valency 2, 4 or 8 and all the faces have valency 3 . Therefore, the resulting maps will have type $\{3,8\}$. These maps are 
also 2-face-colorable, since all the pieces are 2-face-colourable and each of their boundary components has two edges incident with faces of opposite colors.

Then, if we take the duals of these 2 -face-colourable maps of type $\{3,8\}$ we get maps which are bipartite and of type $\{8,3\}$. Hence, these can be understood as the Walsh bipartite maps for hypermaps of type $(3,3,4)$. The gluing process of the required pieces, in order to get an infinite number of these with any genus, is exactly as before.

\subsection{Hypermaps of type $(3,3, n)$ for even $n \geqslant 6$}

To solve this case we use the same 2 -trisc as in Section 8.1 but this time removing two rectangles given by $0<x<1, n-3<y<n$ and by $2<x<3, n-6<y<n-3$ (see Figure 10). Consequently, not all the vertices in the boundary components have the same valency, so we need to explicitly write the type of those boundary components. They are cycles of length 8 and type $(2,2,3,3,2,2,3,3)$ in cyclic order. Both of them are of this type but if we fix an orientation, travelling around each component in the same direction, the first colour of these vertices is black in one of the boundary components and white in the other one. Hence, after the second consecutive vertex of order 2 we will have a black vertex of order 3 in one boundary and a white vertex of order 3 in the other one. Those two boundary components of the same type will be called, respectively: black component and white component.

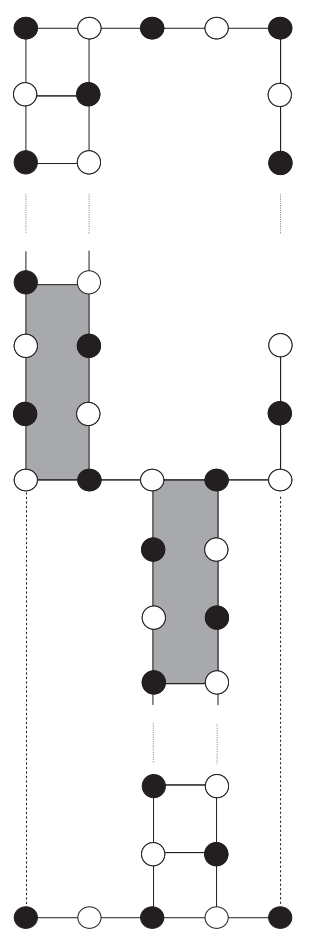

Figure 10: 2-trisc map with boundary components of type $(2,2,3,3,2,2,3,3)$. 
To build the map on the annulus we use two copies of the rectangle $R_{2}$ of the first case, one for $0 \leqslant x \leqslant 4$ and another for $4 \leqslant x \leqslant 8$, identifying the two sides of this bigger rectangle. It has boundary components for $y=0$ and $y=2 n-6$, both of type $(2,2,3,3,2,2,3,3)$, like the map in the 2-trisc, one black and another one white (see Figure 11). Finally, the disc is just an octagon with two edges from two consecutive vertices to

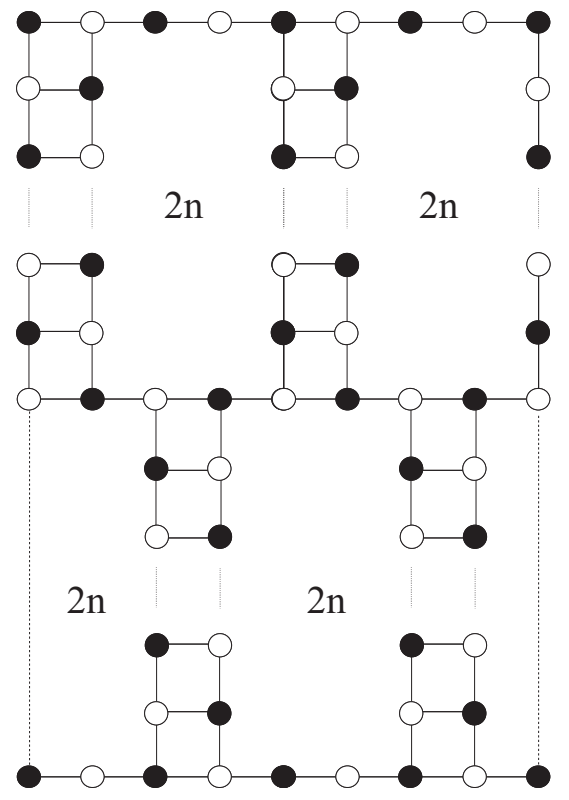

Figure 11: 2-trisc map with boundary components of type $(2,2,3,3,2,2,3,3)$.

their opposite. This gives the disc a white $\left(D_{w}\right)$ or, if we interchange colours, black $\left(D_{b}\right)$ boundary component of the same type $(2,2,3,3,2,2,3,3)$ (see Figure 12). Because all

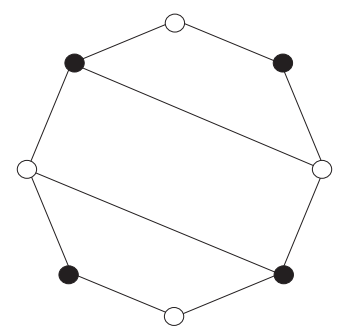

Figure 12: A disc map with a boundary component of type $(2,2,3,3,2,2,3,3)$.

the internal vertices are trivalent, and all faces are of order dividing $2 n$ we will obtain hypermaps of the correct type by conveniently gluing the pieces:

- a black boundary is joined to a white boundary;

- a vertex of valency 2 is identified with another of valency 3 . 


\section{Hypermaps of type $(m, m, n+1)$ with $n+1$ odd: the general method}

If $m, n+1 \geqslant 5$ we can use the construction presented for $n$ even and introduce some slight but important changes. In the previous cases we could use faces of valency 4 in our pieces because these would correspond to hyperfaces of valency 2 , which do not interfere with the type of a hypermap if we require the parameter $n$ (the l.c.m of the valency of the faces) to be even. However, these faces of valency 4 can not appear if we want $n+1$ to be odd. Therefore, other tools must be developed to solve this problem. The general method is the following: to build a hypermap of type $(m, m, n+1), n+1$ odd, we take the pieces that we have built for hypermaps of type $(m, m, n)$ and add new vertices and edges in order to increase by 2 the valency of the old faces of valency $2 n$ and transform all the square faces into faces of valency $2 n+2$.

The first part is easier because we just need a new edge and a new vertex.

For the second, a more delicate procedure, we need to introduce a few stalks of length $n-1$ : paths of length $(n-1)$ with consecutive vertices $v_{0}, v_{1}, \ldots, v_{n-1}$ alternately black and white and with alternate edges $v_{i} v_{i+1}\left(i\right.$ odd) multiplied by $m-1$ so that $v_{0}$ and $v_{n-1}$ have valency 1 while the others have valency $m$.

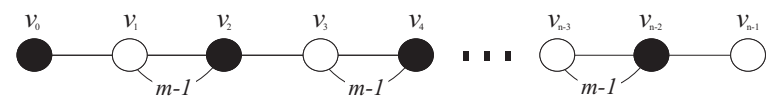

Figure 13: A stalk

By attaching a stalk $S$ to a vertex $v$ within a face $F$ we mean identifying $v_{0}$ or $v_{n-1}$ with $v$, as $v$ is black or white, and embedding the rest of the stalk in $F$ without crossings. This raises the valency of the face $F$ by $2(n-1)$ and that of $v$ by 1 . It also introduces $(m-2)(n-2) / 2$ new faces of valency 2 , together with $n-2$ vertices of valency $m$ and one of valency 1 . Because these new faces have valency 2 they correspond to hyperfaces of valency 1 , so they do not affect the type of the final hypermap. On the other hand, the vertex where the stalk is attached increases its valency by 1 , which means that we need to correct this change by modifying the factors by which certain edges are multiplied. We will describe this operation later with more details.

\subsection{Hypermaps of type $(m, m, n+1)$ with $n+1$ odd with $m \geqslant 5$ and $n+1 \geqslant 5$}

Let $\mathcal{T}_{n}$ be the trivalent bipartite map constructed in section 8.1 by identifying opposite sides of the rectangle $R_{n}$. If we remove, as before, the two square faces given by $0<x<1$, $n-3<y<n-2$ and by $2<x<3, n-4<y<n-3$, the underlying surface is a 2 -trisc. In the face of valency $2 n$ given by $1<x<4, \quad n-3<y<2 n-6$ we insert a white vertex, joined by an edge to the black vertex at $(1, n-3)$, and in the other face of valency 
$2 n$ we insert a black vertex, joined by an edge to the white vertex at $(2, n-3)$, so that both of these faces now have valency $2(n+1)$. At each white vertex of the form $(i, j)=$ $(0, n-1),(0, n+1), \ldots,(0,2 n-7)$ or $(2,1),(2,3), \ldots,(2, n-5)$ we attach a stalk of length $n-1$, with all interior vertices of order $m$, within the incident square face $i<x<i+1$, $j<y<j+1$; at each black vertex of the form $(i, j)=(1, n-1),(1, n+1), \ldots,(1,2 n-7)$ or $(3,1),,(3,3), \ldots,(3, n-5)$ we also attach a stalk of length $n-1$, with all interior vertices of order $m$, within the incident square face $i-1<x<i, j-1<y<j$. The result of this is that each of the $2 n-8$ originally square faces now contains a stalk, and hence has valency $2(n+1)$. Since $m \geqslant 5$, we can choose integers $m_{0} \geqslant 4$ and $m_{1} \geqslant 3$ so that $m_{0}+m_{1}=m+2$ and then multiply the horizontal boundary edges $0<x<1, y=n-3$ and $0<x<1, y=n-2$ by $m_{0}-3$ and $m_{0}-2$, respectively, and the other two horizontal boundary edges $2<x<3, y=n-4$ and $2<x<3, y=n-3$ by $m_{1}-2$, so that the boundary components have types $\left(m_{0}-1, m_{0}, m_{0}, m_{0}\right)$ and $\left(m_{1}+1, m_{1}, m_{1}, m_{1}\right)$, with both first vertices of these sequences being white vertices. Finally, we multiply each remaining horizontal internal edge of the form $i<x<i+1, y=j$ for $i=0$ or $i=2$ by $m-2$ or $m-3$ as $i+j$ is even or odd, that is, as the vertex $(i, j)$ is black or white, so that all internal vertices have valency $m$. This map on the 2-trisc is represented in Figure 14 (before multiplication of edges), with stars representing the stalks of length $n-1$.

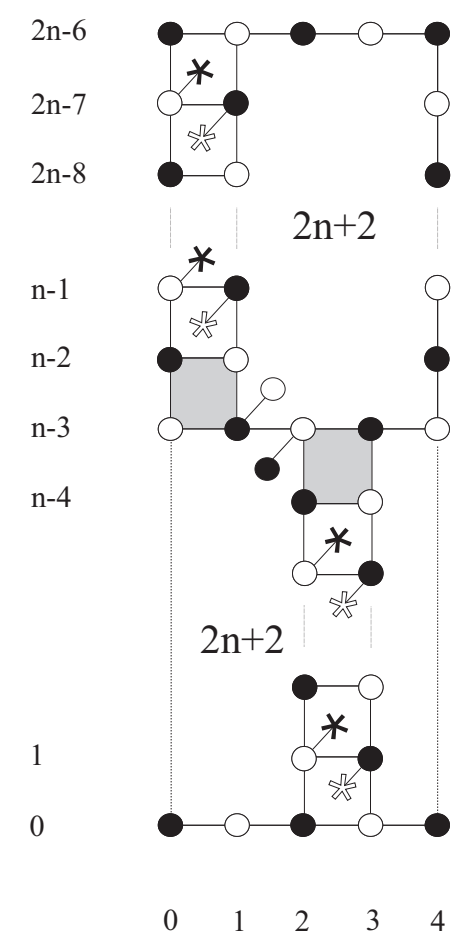

Figure 14: 2-trisc map for the odd case (before multiplication of edges).

To construct a map $\mathcal{A}$ on the annulus, before identifying the vertical sides of $R_{n}$, we insert a black vertex in the face of valency $2 n$ given by $1<x<4, \quad n-3<y<$ $2 n-6$, joined by an edge to the white vertex at $(3,2 n-6)$, and in the other face of 
valency $2 n$ we insert a white vertex, this time joined by an edge to the black vertex at $(0,0)$. At each white vertex of the form $(i, j)=(0, n-3),(0, n+1), \ldots,(0,2 n-7)$ or $(2,1),,(2,3), \ldots,(2, n-5)$ we attach a stalk of length $n-1$, with all interior vertices of order $m$, within the incident square face $i<x<i+1, j<y<j+1$; at each black vertex of the form $(i, j)=(1, n-1),(1, n+1), \ldots,(1,2 n-7)$ or $(3,1),,(3,3), \ldots,(3, n-3)$ we also attach a stalk of length $n-2$, with all interior vertices of order $m$, within the incident square face $i-1<x<i, j-1<y<j$. It follows that each of the $2 n-6$ square faces now has valency $2(n+1)$. This annulus is represented in Figure 15 (before multiplication of edges), with stars representing the stalks of length $n-1$.

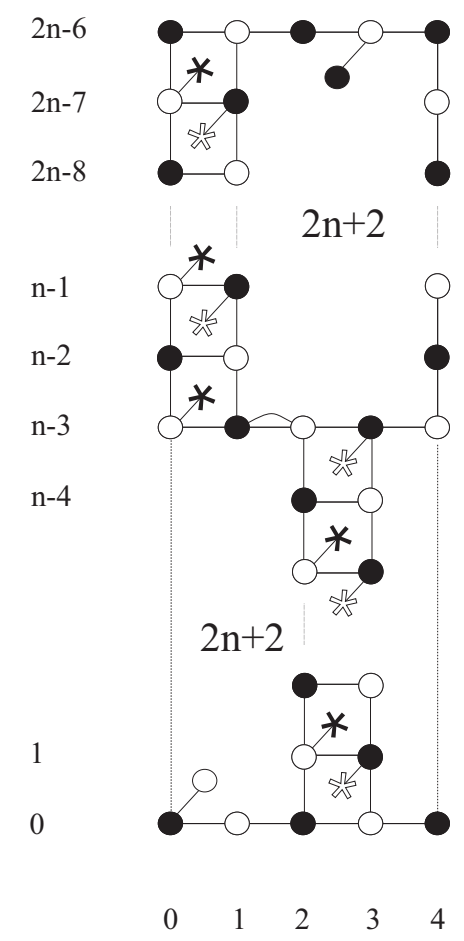

Figure 15: Annulus map for the odd case (before multiplication of edges).

We then multiply the boundary edges $0<x<1, y=0$ and $2<x<3, y=0$ by $m_{0}-2$, and the boundary edges $0<x<1, y=2 n-6$ and $2<x<3, y=2 n-6$ by $m_{1}-2$ and $m_{1}-1$, respectively, so that the two boundary components $y=0$ and $y=2 n-6$ have types $\left(m_{0}-1, m_{0}, m_{0}, m_{0}\right)$ and $\left(m_{1}+1, m_{1}, m_{1}, m_{1}\right)$, with both first vertices of these sequences being white vertices. Finally we multiply each horizontal internal edge $i<x<i+1, y=j$ for $i=0$ or $i=2$ by $m-2$ or $m-3$ as $i+j$ is even or odd and we add an extra edge between vertices $(1, n-3)$ and $(2, n-3)$, so that all internal vertices have valency $m$.

Finally, we will need two discs. To build the disc $\mathcal{D}_{a}$ we construct a tessellation $D_{a}$ of a closed disc $D$, with boundary type $\left(m_{1}+1, m_{1}, m_{1}, m_{1}\right)$. We achieve this by starting with a square, regarded as a bipartite map on $D$ with one face and with four vertices and four edges on $\delta \mathcal{D}$. We multiply each pair of opposite edges by $m_{1}-3$, introducing $2\left(m_{1}-4\right)$ extra faces of valency 2 , so that all four vertices have valency $m_{1}$. Then we introduce, 
within the face of valency 4 , a stalk of length $n-1$ starting at a white vertex. We will get a disc with $2\left(m_{1}-4\right)$ internal faces of valency 2 , one face of valency $2 n+2$ and with a boundary component of type $\left(m_{1}+1, m_{1}, m_{1}, m_{1}\right)$, with the first vertex of this sequence being white (see Figure 16(a)). For the other disc, $\mathcal{D}_{b}$, we use the same tessellation but with a stalk at a black vertex, and instead of multiplying both opposite edges by $m_{0}-3$ we multiply just one of them by $m_{0}-3$ (the one that is not adjacent to the black vertex with a stalk) and the other by $m_{0}-4$. Then, we will get a disc with $2\left(m_{0}-4\right)$ internal faces of valency 2 and one face of valency $2 n+2$, with a boundary component of type $\left(m_{0}-1, m_{0}, m_{0}, m_{0}\right)$, with the first vertex of this sequence being also white (see Figure $16(\mathrm{~b}))$.

(a)

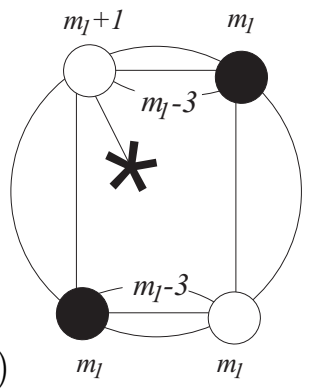

(b)

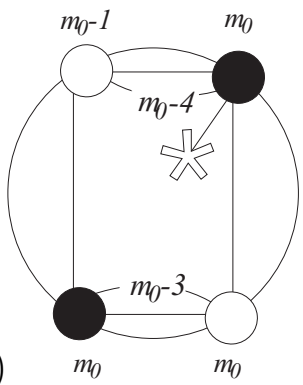

Figure 16: (a) Disc a. (b) Disc $b$.

Using the bipartite map $\mathcal{A}$, together with $\mathcal{B}, \mathcal{D}_{a}$ and $\mathcal{D}_{b}$, the proof proceeds as in earlier cases though we have to be careful, this time, to always attach the boundary white vertices of valency $m_{0}-1$ to the boundary white vertices of valency $m_{1}+1$.

\subsection{Hypermaps of type $(m, m, 3)$ with $m \geqslant 5$}

To build $\mathcal{A}$ we take the rectangle $[0,1] \times[0,6] \subset \mathbb{R}^{2}$, tesselated by six squares. The vertices, as in previous examples, are the integer points $(i, j)$, coloured black or white as $i+j$ is even or odd, joined by edges along the sides and from $(0, j)$ to $(1, j)$ for $j=1, \ldots, 5$. Before identifying the vertical sides $y=0$ and $y=6$, we multiply three of the vertical edges: $x=1,1 \leqslant y \leqslant 2,3 \leqslant y \leqslant 4$ and $5 \leqslant y \leqslant 6$ by $m-4$. This means that in one of the sides we are multiplying alternate edges leaving the other ones unaltered. To increase the valency of the faces and make them of valency 6 , corresponding to hyperfaces of valency 3 , we also need to add a stalk of length 1 (which is just an edge with a vertex) at each of the six vertices with $x=1$ (the ones on the right side of the rectangle), in the 4-gonal face below and to the left of those vertices. Thus, we get six faces of valency 6 and the rest of valency 2, as it can be verified with the help of Figure 17. All the six internal vertices are of valency 1 and, because of that, they do not interfere with the type of our final hypermap.

On the other hand, the boundary components $x=0$ and $x=1$ have types $3^{(6)}$ and $(m-1)^{(6)}$, respectively. 


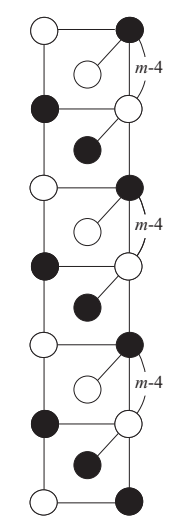

Figure 17: Annulus map for hypermaps of type $(m, m, 3)$ with $m \geqslant 5$.

To construct $\mathcal{T}$ we take another rectangle, this time the rectangle $[0,2] \times[0,8] \subset \mathbb{R}^{2}$, with opposite sides identified. The vertices are again at the integer points $(i, j)$, coloured black or white as $i+j$ is even or odd. There are vertical edges between $(i, j)$ and $(i, j+1)$ for $i=0,1,2$ and $j=0, \ldots, 7$ with those between $(i, 1)$ and $(i, 2)$, for $i=0,1$, multiplied by $m-3$. The horizontal edges are the ones between $(i, j)$ and $(i+1, j)$ for $i=0$ and $j$ even, and for $i=1$ and $j$ odd. Some of these, the ones between $(1, j)$ and $(2, j)$ for $j=3$ and 7 , are multiplied by $m-2$ and the edge between $(0,0)$ and $(1,0)$ is multiplied by $m-3$. We then remove two 6 -gonal faces, the ones given by $0<x<1$ for $0<y<2$ and $4<y<6$. This will leave us with boundary components of types $(m-1)^{(6)}$ and $3^{6}$ respectively (see Figure 18).

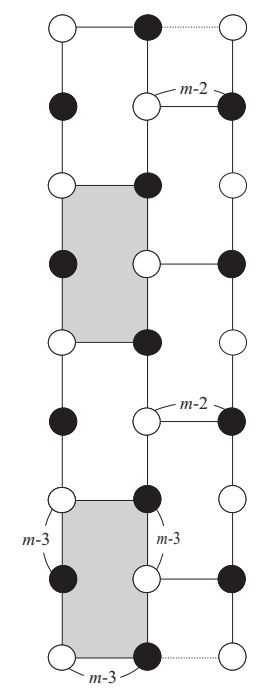

Figure 18: 2-trisc map for hypermaps of type $(m, m, 3)$ with $m \geqslant 5$.

Six of the faces of $\mathcal{T}$ are 6 -gons and the rest are 2 -gons. All the four internal vertices 
are of valency $m$, which is important to obtain the required final type.

Finally, for the disc we need to place six vertices around the boundary of $D$, alternately black and white. Three alternate boundary segments are multiplied by $m-2$ and by this process all the six vertices have valency $m-1$ and the disc has type $(m-1)^{(6)}$. All the faces of this piece have valency 2 except one of them that has valency 6 (see Figure 19).

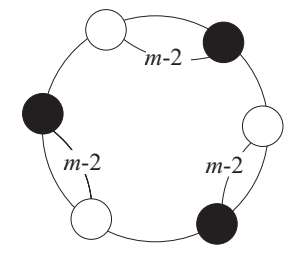

Figure 19: Disc map for hypermaps of type $(m, m, 3)$ with $m \geqslant 5$.

We also need another disc of type $3^{(6)}$. That can be achieved by multiplying alternate boundary segments by 2 instead of $m-2$. To obtain the final hypermaps we just need to join the boundary components of type $(m-1)^{(6)}$ with those of type $3^{(6)}$, proceeding as in earlier cases.

\subsection{Hypermaps of type $(4,4,3)$}

This case must be dealt separately because the previous annulus does not work for such a low value for $m$. If $m=4, m-4$ would be 0 and that will lead us to a nonconnected graph. However, we can use the same $\mathcal{T}$ and $\mathcal{D}$ as in the previous section and proceed as before, in earlier proofs. The new annulus is the following: we take a rectangle $[0,2] \times[0,6] \subset \mathbb{R}^{2}$, with vertices at the integer points $(i, j)$, colored black or white as $i+j$ is even or odd. The edges are along the sides, and also from $(i, j)$ to $(i+1, j)$, for $i=0,1$ and $j=0, \ldots, 6$, so that the rectangle is tessellated by six faces, all of valency 6 (see Figure 20).

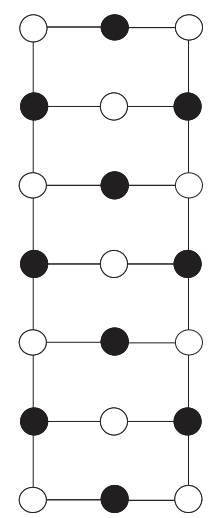

Figure 20: Annulus map for hypermaps of type $(4,4,3)$. 
The side $y=0$ is identified with the side $y=6$ and the two boundary components are both of type $3^{(6)}$.

\subsection{Hypermaps of type $(4,4, m)$, odd $m \geqslant 5$}

If, by a Machi operation [5], we transpose hyperedges and hyperfaces, we will deal with hypermaps of type $(4, m, 4)$ instead, which will be enough to solve this case.

To construct the 2 -trisc we take the rectangle $[0,3] \times[0,2] \subset \mathbb{R}^{2}$, with vertices at the integer points $(i, j)$ coloured black or white as $i+j$ is even or odd. The edges are around the sides and also from $(1,1)$ to $(0,1),(1,0),(2,1)$ and $(1,2)$, and from $(2,1)$ to $(2,2)$. We then remove the square $1<x<2,1<y<2$.

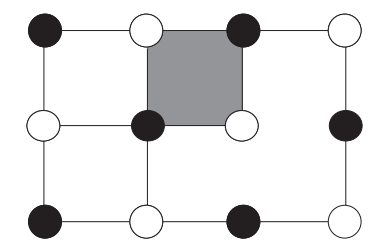

Figure 21: 1-trisc map.

The identifications of the sides of the rectangle are slightly different from previous cases: the side $y=0$ is identified with the side $y=2$ by putting $(x, 0)=(x, 2)$ and the side $x=0$ is identified with the side $x=3$ by putting $(0, y)=(3, y+1)$ where we take $y+1$ mod (2). Within the square $0<x<1,1<y<2$ we draw $m-5$ paths of length 2 between the black vertices at $(0,2)$ and $(1,1)$, each containing a white vertex of valency 2 , creating $(m-5) / 2+2$ new faces of valency 4 . Then, in one of the new faces that shares edges with the old one, we draw two paths of length 1 , each joining the black vertex at $(0,2)$ to another white vertex (see example on Figure 22). This last step creates a new face of valency 8 , representing a hyperface of valency 4). By this process we obtain a torus

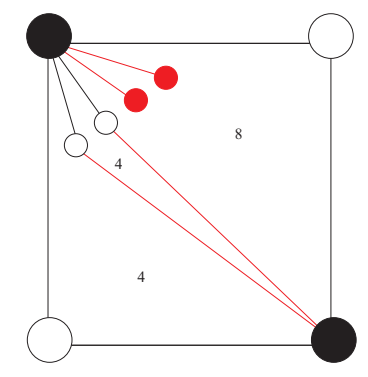

Figure 22: A face of valency 8 transformed into two faces of valency 4 and one of valency 8.

minus a disc, with boundary having black vertices of order 4 and 2 , and white vertices of 
valencies $m-1$ and 3 . This torus minus one disc (1-trisc) has two 8-gonal faces and the other faces are 4-gons. Its unbranched double covering gives us the 2 -trisc, with twice the number of faces.

To construct the annulus we consider the rectangle $[0,1] \times[0,4] \subset \mathbb{R}^{2}$ with vertices at integer points $(i, j)$ coloured white or black as $i+j$ is even or odd. There are edges around the sides and also from $(0,3)$ to $(1,3)$. The edges from $(0,2)$ to $(0,3)$ and from $(1,0)$ to $(1,1)$ are multiplied by 2 . Within the square $0<x<1,3<y<4$ we draw $m-5$ paths of length 2 between the black vertices at $(0,4)$ and $(1,3)$, each containing a white vertex of valency 2 . From each white vertex $(0,4)(1,3)$, and inside the same face, we draw two paths of length 1 .

Finally we identify the side $y=0$ with the side $y=4$ to form an annulus. Both boundary components have the same type $(m-1,4,3,2)$ as the ones in the 2-trisc but with mutually inverse cyclic orders, so we need to use these annuli in mirror-image pairs to make the identification work properly.

To construct the disc $\mathcal{D}$ we place four vertices around the boundary of $\mathcal{D}$, two black alternating with two white, joined by four edges around the boundary. We then join one black vertex to the two white vertices by edges across the interior, creating one 4-gonal face and two 2-gons. Within one of these 2-gons, we place $k=(m-5) / 2+1$ black vertices, each joined by a pair of edges to the white in nested fashion, creating one 2-gon and $k$ 4-gons (see Figure 23 for an example).

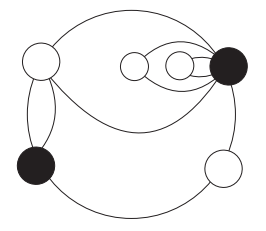

Figure 23: Example of a disc with boundary of type $(2,4,7,2)$.

This will make the boundary of the disc have the same type as the boundary components of the 2-trisc and the annulus. In the gluing process, we need to join vertices of order $m-1,4,3$ and 2 with vertices of order $3,2, m-1$ and 4 , respectively, to get black vertices of order $m$ and white vertices of order 4 .

\subsection{Hypermaps of type $(3,3, m)$, for odd $m \geqslant 5$}

In order to build the required hypermaps of type $(3,3, m)$, for odd $m \geqslant 5$, we will use 2face colourable maps of type $\{3,2 m\}$ and then take the duals of these, a method previously used in Section 8.4 for the case $(3,3,4)$. Let $R$ be the rectangle $[0,6] \times[0,2] \subset \mathbb{R}^{2}$ with vertices at $(i, j)$ (with $i \in\{1,3,5\}$ and $j \in\{0,2\}$ ) and $(i, 1)$ with $i \in\{0,2,4,6\}$ ). There are horizontal edges between all consecutive vertices with the same horizontal coordinates and also:

$$
(i, 1) \times(i-1, j) \text { if } i \in\{2,4,6\} \text { and } j \in\{0,2\}
$$




$$
(i, 1) \times(i+1, j) \text { if } i \in\{0,2,4\} \text { and } j \in\{0,2\}
$$

We then identify opposite sides of the rectangle to get a torus. This will give rise to 11 triangular faces but we remove two of them (one corresponding to the triangle of vertices $(0,1),(1,2),(5,2)$ and the other to the triangle $(2,1),(3,2),(4,1)$. The face $(2,1),(3,0),(4,1)$ is coloured red and the remaining ones are coloured red or white in such a way that no adjacent faces have the same colour (this operation is possible because this map is 2-face colourable). Hence, there are three vertices in each boundary component of the 2-trisc adjacent to three red faces, in one case, and three white faces on the other ${ }^{1}$. All the vertices have valency 6 . To build the right map on the 2 -trisc, we need to add a suitable amount of wedges to some faces (see Figure 24 for an example), before identification.

A wedge $\left(v_{0}, w, v_{1}\right)$, attached to vertices $v_{0}, v_{1}$ on a triangular face $f=\left(v_{0}, v_{1}, v_{2}\right)$, is constructed by adding a vertex $w$ inside the face $f$ and then joining $w$ to $v_{0}$ and $v_{1}$, within $f$, and adding another edge, also within $f$, between $v_{0}$ and $v_{1}$ in such a way that $\left(v_{0}, v_{1}, v_{2}\right)$ is still a triangular face. Each time we introduce a wedge to a face, we are also adding two more triangular faces to the map. And if the original map is 2-face colourable, so it will be after introducing as many wedges as we want.

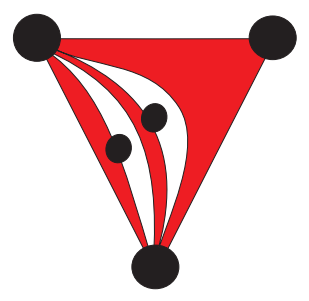

Figure 24: 2 wedges attached to the same vertices and on the same face.

In this case, to build our 2-trisc map, we need to add, three times, $(2 m-10) / 4$ wedges. First, between vertices $(2,1),(3,2)$ inside the face $((2,1),(3,2),(1,2))$, then between vertices $(3,2),(4,1)$ inside the face $((3,2),(4,1),(5,2))$, and finally between vertices $((2,1),(4,1)$ inside the face $((2,1),(4,1),(3,0)$. We will get then two boundary components, one of type $6^{(3)}$ and another one of type $(2 m-4)^{(3)}$ (see Figure 25, an example of a 2-Trisc for $m=7$ ).

To build the annulus we use the same rectangle $[0,6] \times[0,2] \subset \mathbb{R}^{2}$ with the vertices in the same places as before. However we do not remove any triangular face and we attach $(2 m-8) / 2$ wedges to each one of the following two pairs of vertices:

$$
(1,2),(3,2) \text { inside face }((1,2),(3,2),(2,1)),
$$

\footnotetext{
${ }^{1}$ The colours of the faces adjacent to the boundary components are important because we want the final map to be also 2-face colourable and this is only possible if we have no adjacent faces of the same colour after gluing the pieces.
} 


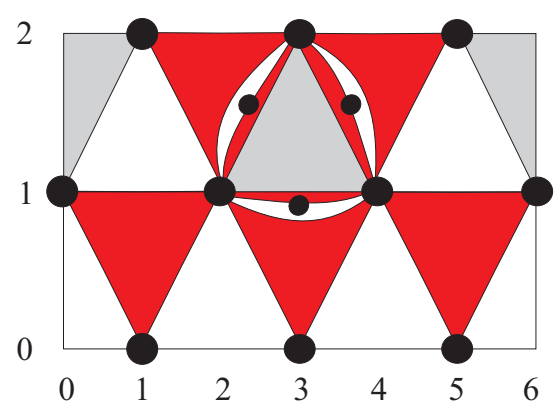

Figure 25: 2-Trisc for $m=7$.

$(6,1),(5,2)$ inside face $((6,1),(5,2),(1,2))$;

$(2 m-6) / 2$ wedges to the following pair of vertices:

$(2,1),(4,1)$ inside face $((2,1),(4,1),(3,2))$;

and one wedge to each of the two following pairs:

$$
\begin{aligned}
& (3,0),(5,0) \text { inside face }((3,0),(5,0),(4,1)), \\
& (0,1),(1,0) \text { inside face }((0,1),(1,0),(2,1))
\end{aligned}
$$

If we identify the vertical sides of the rectangle we get a map on an annulus and, by the way we constructed it, that map is 2-face colourable (we use the opposite colour scheme we have used in the 2-trisc and we use the white colour to each wedge inside a red triangular face, and the red colour to each wedge inside a white triangular face). It follows that one of the boundary components has type $6^{(3)}$ and is adjacent to three red faces, and the other one has type $(2 m-4)^{(3)}$ and is adjacent to three white faces (see Figure 26 for an example). All the interior vertices have valency $2 \mathrm{~m}$.

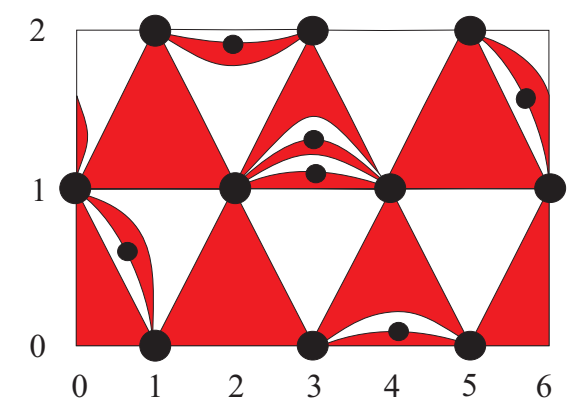

Figure 26: Annulus for $m=5$.

To construct the disc, we take $D=\{z \in \mathbb{C}:|z| \leqslant 2\}$, with vertices at $\pm 2, \pm 1+i$, 0 and $2 i$, with edges along the boundary components and joining the vertex at -2 to 0 
and $-1+i$, the vertex at 2 to 0 and $1+i$, and the vertex at $2 i$ to $1+i$ and $-1+i$. We have then, at this stage, three triangular faces and one face of valency 6 . Inside this hexagonal face we add three more edges, between -2 and 2 , between -2 and $2 i$, and between 2 and $2 i$. Hence, this new Disc, $D_{1}$, has 7 triangular faces, is 2 -face-colourable and has type $6^{(3)}$ (see Figure 27). Depending on the way we coloured the faces, we might have three white faces adjacent to the boundary component or three red faces instead. If we introduce $(2 m-6) / 4$ wedges attached to each one of the three possible different pairs of boundary vertices (and in each one of the three boundary faces) we will get again a 2 -face-colourable disc, $D_{2}$, but this time of type $(2 m-4)^{(3)}$.

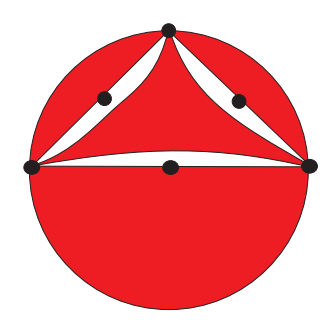

Figure 27: Disc $D_{1}$ with red boundary.

To build infinitely many hypermaps of type $(3,3, m)$ we need to glue, a suitable number of times, the red boundary component $6^{(3)}$ of an annulus (or a 2-trisc, or a disc) with the white boundary $(2 m-4)^{(3)}$ of another annulus (or a 2-trisc, or a disc).

This completes the proof of Theorem 1.1.

\section{Reduction to the planar case}

A 2-sheeted covering of the sphere, branched over four points, is a torus. A 2-sheeted covering of a torus, branched over two points, is a surface of genus 2 . If $g \geqslant 3$ then a $(g-1)$-sheeted unbranched covering of a surface of genus 2 is a surface of genus $g$.

Now suppose that we can construct infinitely many hypermaps of type $(l, m, n)$ and genus 0 , where one of $l, m, n$ (say $m$ ) is even, and each hypermap has at least five hyperedges $e_{1}, \ldots, e_{5}$ of valency dividing $m / 2$. Taking 2 -sheeted coverings branched over $e_{1}, \ldots, e_{4}$ gives infinitely many hypermaps of type $(l, m, n)$ and genus 1 , each with at least two hyperedges $e_{5}^{\prime}$ and $e_{5}^{\prime \prime}$ (covering $e_{5}$ ) of valency dividing $m / 2$. Taking 2 -sheeted coverings of these branched over $e_{5}^{\prime}$ and $e_{5}^{\prime \prime}$ gives infinitely many hypermaps of type $(l, m, n)$ and genus 2. Taking $(g-1)$-sheeted unbranched coverings of these gives infinitely many hypermaps of type $(l, m, n)$ and genus $g \geqslant 3$.

In particular, taking $m=2$, if we can construct infinitely many planar maps of a given type, each with at least five free edges, then we can construct infinitely many maps of genus $g$ of that type for each $g$. 


\section{Maps of type $\{3,7\}$.}

One of the most interesting of the hyperbolic triangle groups is $\Delta=\Delta(2,3,7)$ : for example, the normal subgroups of finite index in $\Delta$ uniformise those compact Riemann surfaces of genus $g>1$ which attain Hurwitz's upper bound of $84(g-1)$ automorphisms. By contrast, the subgroups of finite index we are interested in here are generally not normal. By earlier remarks, if we regard $\Delta$ as the triangle group $\Delta(7,2,3)$ then conjugacy classes of subgroups of finite index in $\Delta$ correspond to compact oriented hypermaps of type $(7,2,3)$, that is, compact oriented maps of type $\{3,7\}$ in Coxeter and Moser notation [2], with the subgroups having the same genus as the map. As a simple example of the more general methods to be used later, we will prove the following result:

Theorem 11.1. For each integer $g \geqslant 0$ there are infinitely many nonisomorphic maps of type $\{3,7\}$ and genus $g$, including at least one uniform map if $g \geqslant 2$.

As an immediate corollary we have:

Corollary 11.1. For each integer $g \geqslant 0$ the triangle group $\Delta=\Delta(2,3,7)$ has infinitely many subgroups of finite index and of genus $g$, including at least one surface subgroup if $g \geqslant 2$.

Proof of Theorem 11.1: Our method is to construct maps of type $\{3,7\}$ by joining together suitable numbers of copies of three basic 'Lego' pieces, each carrying a triangulation. These are a $\operatorname{disc} \mathcal{D}$, an annulus $\mathcal{A}$, and a pair of pants $\mathcal{P}$ (topologically a sphere minus one, two or three open discs).

We form $\mathcal{P}$ by subdividing a pair of pants $P$ into 14 triangles, with no vertices in the interior of $P$, so that each of its three boundary components is a 4-gon with its edges as part of the triangulation, and with vertices of valencies 4,5,4 and 5 in cyclic order. For instance one can construct $\mathcal{P}$ by choosing three pairs of adjacent faces of an icosahedron, no two pairs having a vertex in common, and removing these six faces together with the three edges separating the faces in each pair.

We take $\mathcal{D}$ to be the closed unit disc $D$ in $\mathbf{C}$, with four edges around the boundary $\partial D=S^{1}$ joining vertices at \pm 1 and $\pm i$, and three disjoint edges across $D$ joining the pairs of vertices $-i$ and 1,1 and -1 , and -1 and $i$, so that $D$ is subdivided into two faces of valency 3 and two of valency 2 . We then add a loop at each of the vertices $\pm i$ within its incident face of valency 2, dividing this face into two faces of valencies 1 and 3 (see Figure 28 ). Thus $\mathcal{D}$ has four faces of valency 3 and two of valency 1 , and its boundary is a cycle of length 4 , with vertices of valencies $4,5,4$ and 5 in that cyclic order. We construct $\mathcal{A}$ from $\mathcal{P}$ by capping one of its three 'holes' with the disc $\mathcal{D}$. If we identify $\partial \mathcal{D}$ with one of the boundary components of $\mathcal{P}$, so that vertices of valency 4 are matched with those of valency 5 , the result is an annulus $\mathcal{A}$ subdivided into 18 faces of valency 3 and two of valency 1 ; it has four internal vertices of valency $4+5-2=7$ on $\partial D$, and eight boundary vertices (those of $\partial \mathcal{P} \backslash \partial \mathcal{D}$ ), four each of valencies 4 and 5 .

If $g \geqslant 1$ and $k \geqslant 0$, with $k \geqslant 1$ if $g=1$, we take $2(g-1)$ copies of $\mathcal{P}$ and $k$ copies of $\mathcal{A}$. By making suitable pairwise identifications of their $6(g-1)+2 k$ boundary components, 


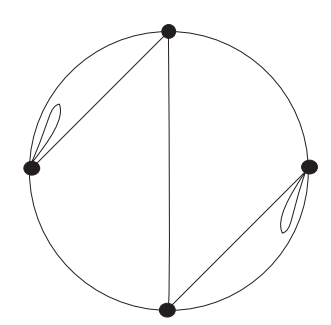

Figure 28: Disc.

with vertices of valency 4 matched with those of valency 5 , one can construct a compact orientable map $\mathcal{M}_{g, k}$ and genus $g$. This map is without boundary, and its vertices all have valency 7 ; in addition to its $28(g-1)+18 k$ faces of valency 3 it has $2 k$ of valency 1 (two on each copy of $\mathcal{A}$ ), so it corresponds to a subgroup $M_{g, k}$ of signature $\left(g ; 3^{(2 k)}\right)$ and index $84(g-1)+56 k$ in $\Delta$. In particular, if $g \geqslant 2$ then by taking $k=0$ we obtain a uniform map $\mathcal{M}_{g, 0}$, corresponding to a surface group of genus $g$ and index $84(g-1)$ in $\Delta$.

The construction for $g=0$ is similar, but we now use $k$ copies of $\mathcal{A}$ and two of $\mathcal{D}$, where $k \geqslant 0$, to obtain a spherical map of type $\{3,7\}$ with $18 k+8$ faces of valency 3 and $2 k+4$ of valency 1 , corresponding to a subgroup of signature $\left(0 ; 3^{(2 k+4)}\right)$ and index $56 k+28$ in $\Delta$.

\section{References}

[1] M. D. E. Conder, Generators for alternating and symmetric groups, J. London Math. Soc. (2) 22 (1980), no. 1, 75-86.

[2] H. S. M. Coxeter, W. O. J. Moser, Generators and Relations for Discrete Groups, 2nd ed, Springer-Verlag (1965).

[3] G. A. Jones, Triangular maps and noncongruence subgroups of the modular group, Bull. London Math. Soc. 11 (1979), 117-123.

[4] G. A. Jones and D. Singerman, Maps, hypermaps and triangle groups, in The Grothendieck Theory of Dessins d'Enfants (L. Schneps ed.), London Math. Soc. Lecture Note Ser. 200 (1994), 115-145.

[5] A. Machì, On the complexity of a hypermap, Discrete Math. 42 (1982), 221-226.

[6] D. Pinto, Hypermaps: constructions and operations, $\mathrm{PhD}$ thesis, University of Southampton (2009).

[7] D. Singerman, Finitely maximal Fuchsian groups, J. London Math. Soc. (2) 6 (1972) 29-38.

[8] W. W. Stothers, Subgroups of the $(2,3,7)$ triangle group, Manuscripta Math. 20 (1977), 323-334.

[9] T. R. S. Walsh, Hypermaps versus bipartite maps, J. Combinatorial Theory Ser. B 18 (1975), 155-163. 